\title{
Publicly available machine learning models for identifying opioid misuse from the clinical notes of hospitalized patients
}

Brihat Sharma ${ }^{1}$, Dmitriy Dligach ${ }^{1,2}$, Kristin Swope ${ }^{3}$, Elizabeth Salisbury-Afshar ${ }^{4}$, Niranjan S. Karnik ${ }^{5}$, Cara Joyce ${ }^{2,3}$ and Majid Afshar $2,6,7^{*}$ (D)

\begin{abstract}
Background: Automated de-identification methods for removing protected health information (PHI) from the source notes of the electronic health record (EHR) rely on building systems to recognize mentions of PHI in text, but they remain inadequate at ensuring perfect PHI removal. As an alternative to relying on de-identification systems, we propose the following solutions: (1) Mapping the corpus of documents to standardized medical vocabulary (concept unique identifier [CUI] codes mapped from the Unified Medical Language System) thus eliminating PHI as inputs to a machine learning model; and (2) training character-based machine learning models that obviate the need for a dictionary containing input words/n-grams. We aim to test the performance of models with and without PHI in a use-case for an opioid misuse classifier.
\end{abstract}

Methods: An observational cohort sampled from adult hospital inpatient encounters at a health system between 2007 and 2017. A case-control stratified sampling $(n=1000)$ was performed to build an annotated dataset for a reference standard of cases and non-cases of opioid misuse. Models for training and testing included CUI codes, character-based, and n-gram features. Models applied were machine learning with neural network and logistic regression as well as expert consensus with a rule-based model for opioid misuse. The area under the receiver operating characteristic curves (AUROC) were compared between models for discrimination. The HosmerLemeshow test and visual plots measured model fit and calibration.

Results: Machine learning models with CUI codes performed similarly to n-gram models with PHI. The top performing models with AUROCs $>0.90$ included CUI codes as inputs to a convolutional neural network, max pooling network, and logistic regression model. The top calibrated models with the best model fit were the CUIbased convolutional neural network and max pooling network. The top weighted CUI codes in logistic regression has the related terms 'Heroin' and 'Victim of abuse'.

(Continued on next page)

\footnotetext{
* Correspondence: Majid.afshar@lumc.edu

${ }^{2}$ Center for Health Outcomes and Informatics Research, Loyola University

Chicago, 2160 S. First Avenue, Maywood, IL 60156, USA

${ }^{6}$ Department of Health Informatics and Data Science, Loyola University

Chicago, Maywood, IL, USA

Full list of author information is available at the end of the article
}

C The Author(s). 2020 Open Access This article is licensed under a Creative Commons Attribution 4.0 International License, which permits use, sharing, adaptation, distribution and reproduction in any medium or format, as long as you give appropriate credit to the original author(s) and the source, provide a link to the Creative Commons licence, and indicate if changes were made. The images or other third party material in this article are included in the article's Creative Commons licence, unless indicated otherwise in a credit line to the material. If material is not included in the article's Creative Commons licence and your intended use is not permitted by statutory regulation or exceeds the permitted use, you will need to obtain permission directly from the copyright holder. To view a copy of this licence, visit http://creativecommons.org/licenses/by/4.0/ The Creative Commons Public Domain Dedication waiver (http://creativecommons.org/publicdomain/zero/1.0/) applies to the data made available in this article, unless otherwise stated in a credit line to the data. 
(Continued from previous page)

Conclusions: We demonstrate good test characteristics for an opioid misuse computable phenotype that is void of any PHI and performs similarly to models that use PHI. Herein we share a PHI-free, trained opioid misuse classifier for other researchers and health systems to use and benchmark to overcome privacy and security concerns.

Keywords: Opioid misuse, Heroin, Opioid use disorder, Natural language processing, Machine learning, Computable phenotype

\section{Background}

Clinical notes from the EHR are promising for modeling prediction tasks in healthcare but they contain protected health information (PHI) and require legal and regulatory approvals to share across hospitals for implementation. The majority of published models that use machine learning for text classification are word-based classifiers that internally store a vocabulary of input features (e.g. word n-grams) [1-4]. Major challenges remain in privacy and security of PHI-laden models for sharing, and it prevents the deployment of NLP models at other hospitals.

Training NLP models in a way that prevents PHI leakage requires a prior step to manually or automatically scrub notes, which can be a laborious process and may not occur at all hospitals. These steps include employing software previously trained to identify and remove individual mentions of PHI [5-8]. Most software were trained on specific types of clinical documents, and many used pattern matching with rules and dictionaries and, more recently, machine learning. These systems do not have perfect accuracy so training machine learning algorithms with features derived from these systems may still lead to PHI embedded in the machine learning model. There remains a paucity of evidence examining approaches that can process and train an entire corpus of text documents in a PHI-free manner.

In this study, we experiment with the following solutions using feature engineering to provide PHI-free models for text classification: (1) using raw text converted into standardized medical vocabulary (concept unique identifiers [CUIs] mapped from the Unified Medical Language System); and (2) character-based models. CUI-based classifiers are PHI-free because their input features are concepts mapped to the Unified Medical Language System as standardized codes (e.g. 'heroin' is mapped to CUI code 'C0011892') that may be deployed across hospitals without concern for PHI leakage. A character-based model uses characters rather than words or n-grams as basic units of input; a classifier's task in this case is to identify salient character sequences that are useful for the prediction task. Like CUI-based models, character-based models are PHI-free since their vocabulary consists of individual unique characters observed in the corpus of text.
Opioid misuse is a behavioral condition that represents a heterogeneous pattern of use rendering it complex to identify from the EHR and is an ideal use-case to examine our PHI-free approach. Patients with opioid misuse represent a vulnerable population so removing PHI for publicly available models is a priority. Opioid misuse is taking an opioid for reasons other than prescribed or as an illicit drug $[9,10]$. Traditionally, diagnostic billing codes or rule-based models for opioid misuse have been used by health systems for health surveillance and monitoring of outcomes [11]. However, International Classification of Diseases- 9 or-10 codes (ICD 9/10) are typically constrained by poor sensitivity/recall with a high false negative rate $[12,13]$. Computable phenotypes that use supervised machine learning may learn the complexities of these behavioral conditions from the clinical notes to predict cases of opioid misuse.

We aim to compare the performance of multiple text classification approaches, including both PHI-laden and PHI-free, for an opioid misuse computable phenotype at a large, tertiary health system using routinely available EHR notes of hospitalized patients. A variety of machine learning models, including multiple neural network architectures that can contain hidden layers with PHI will be examined against our PHI-free approaches. We hypothesize that it is possible to build a PHI-free model for text classification of opioid misuse without sacrificing performance.

\section{Methods \\ Population and setting}

Loyola University Medical Center (LUMC) is a 559-bed hospital and tertiary academic center including a burn and Level 1 trauma center serving Chicago and its western suburbs. LUMC has maintained Epic (Epic Systems Corporation, Verona, Wisconsin) as its EHR vendor since 2003 and includes a Microsoft SQL server-based clinical data warehouse that has been available for research since 2007. The hospital cohort is comprised of 161,520 adult inpatient encounters ( $\geq 18$ years of age) between January 1, 2007 and September 30, 2017. Patients with only outpatient encounters were excluded.

\section{Sampling of hospital cohort to build reference dataset for opioid misuse computable phenotype}

The National Survey on Drug Use and Health (NSDUH) and the National Institute of Drug Abuse (NIDA) define 
opioid misuse as individuals taking an opioid for reasons other than prescribed or as an illicit drug $[9,10]$. Opioid misuse is a behavioral condition that represents a heterogeneous pattern of use ranging from nonmedical prescription drugs to injection illicit drug use rendering it complex to identify from the EHR. To train and test our computable phenotype for opioid misuse, a random case-control sampling of 1000 patients was annotated from the hospital cohort during the study period for chart review. The study cohort for annotation included an oversampling of hospitalizations that had an ICD-9/ 10 codes for opioid-related hospitalizations or a positive urine drug screen for an opioid drug [14]. Additional sampling of at-risk patients included those who had ICD codes for chronic pain, naloxone (reversal drug for opioid overdose) order and administration, or a physician order for a urine drug screen. Age- and sex-matched controls without any of the above criteria were included as potential controls. A trained annotator (KS) performed review of each patient record to provide a final annotation for the likelihood of opioid misuse on a Likert scale (1-5). The annotator met an inter-rater reliability of Cohen's kappa coefficient $\geq 0.75$ with a critical care physician and substance use researcher (MA and ESA) before independent review was continued.

The Likert scale included definite, highly probable, probable, definitely not, and uncertain for determining opioid misuse in accordance with NIDA and NSDUH definitions. Probable cases required any one of the following: (1) history of opioid misuse evident in the clinical notes but no current documentation for the encounter; (2) provider mention of aberrant drug behavior; (3) evidence of other drug misuse (except alcohol) in addition to prescription opioid use. Highly probable cases were classified by more than one of the probable case criteria, or provider mention of opioid dependence plus suspicion of misuse in the clinical notes. Definite cases were classified as the patient self-reporting opioid misuse to a provider or documentation by provider of patient misusing an opioid. For the classification task of the computable phenotype, patients were categorized as exhibiting opioid misuse if they met probable, highly probably, or definite criteria - these were aligned with the definitions by NSDUH and NIDA. The remainder of cases of definitely not or uncertain were categorized as no opioid misuse. Only $1.9 \%(n=19)$ of cases were classified as uncertain. The final reference dataset was comprised of $33.7 \%(n=337)$ cases of opioid misuse.

\section{Rule-based opioid misuse computable phenotype for comparison to machine learning models}

First, a simpler baseline rule-based model was built from structured data for comparison to machine learning models. The rules were developed by substance use specialists including an addiction specialist (ESA) and psychiatrist (NK), and in accordance with the NSDUH and NIDA definition for opioid misuse $[9,10]$. The rulebased criteria for opioid misuse were met if any of the following structured data elements qualified from the EHR: (1) positive urine drug screen for an opioid with cosubstance use with any of the following: an illicit drug (phencyclidine or cocaine), a benzodiazepine that was not self-reported by the patient as a prescribed medication, or an amphetamine that was not self-reported by the patient as a prescribed medication; (2) positive urine drug screen for an opioid only but not self-reported by the patient as a prescribed medication; (3) ICD-9/10 codes for opioid poisoning or intoxication [14].

\section{Machine learning models: PHI-free and PHI-laden}

We experimented with several classes of models to learn the relationship between the input text and the classification task (opioid misuse vs. no opioid misuse) including linear classifiers and several neural network architectures. Encounter-level analysis $(n=1000)$ was performed by incorporating all clinical documents from each patient hospitalization for training the machine learning models.

PHI-laden models: We utilized n-gram classifiers mainly for comparison to PHI-free models (CUIs and characterbased). A logistic regression model and convolutional neural network were trained with n-grams. The n-gram features were examined as unigrams, bigrams, and the combination of the two for the linear model and a 300dimension word embedding for the neural network architectures. We also investigated the possibility of removing PHI from the n-gram models by training a token-based L1-regularized logistic regression model. Unlike L2regularized models that often contain thousands of features, the sparsity of L1-regularized model allows manual examination of the features with non-zero weights to allow for manual removal of PHI-containing features post-hoc.

PHI-free models: We explored both CUI and characterbased CNN models. Linguistic processing of the clinical documents into CUI codes was performed in clinical Text and Knowledge Extraction System (http://ctakes.apache. org) [15]. Named entity mentions in the raw text were mapped to the Unified Medical Language System (UMLS), which includes over 2 million concepts from nearly 9 million distinct names that are merged into the National Library of Medicine's Metathesaurus. The spans of the UMLS named entity mentions (diseases, symptoms, anatomy, and procedures) were mapped from the raw notes and organized into Concept Unique Identifiers (CUIs). The free text in clinical documents from the EHR are matched to a dictionary of concepts, by default SNOMED $\mathrm{CT}$ and RxNORM, and tagged with concept codes from the original dictionary, and a CUI code from the UMLS Metathesaurus. This dictionary lookup puts text into a coded format that completely de-identifies to structured 
data as SNOMED CT and RxNORM CUI codes. For instance, the named entity mention for 'heroin abuse' has the CUI code 'C0600241'. Each named entity mention was also analyzed to determine its negation status (e.g. 'no heroin abuse'). The original cTAKES publication demonstrates that concept mapping and negation status had F1 scores of 0.957 and 0.943 on certain tasks, respectively [15]. Figure 1 represents an example of a $\mathrm{CNN}$-based model for an opioid misuse computable phenotype.

The dataset was split into training $(60 \%)$, validation (20\%), and test (20\%) data. The training set was used for training models, the validation set for parameter tuning, and the test set for the final performance evaluation. Term frequency-inverse document frequency (tf-idf) was applied to normalize the n-gram and CUI codes prior to being fed into the logistic regression models. The neural network architectures considered included: (1) a convolutional neural network (CNN) [13]; (2) a deep averaging network ( [16]; (3) a max pooling network [17]; and (4) combination of deep averaging and max pooling network. The CUI-based CNN includes 1024 filters of size 1. The filter of size one is used because the ordering of CUI codes in clinical texts is typically not meaningful and does not warrant larger filter sizes. A deep averaging network is a simple neural architecture that accepts word embeddings as inputs and averages them. The averaged values are then fed into a classification layer (sigmoid or softmax). The deep averaging network was first introduced in 2015 [17] and later re-introduced in 2016 [18]. This architecture can often be as effective as more complex models such as $\mathrm{CNN}$ or recurrent neural network [19]. A max pooling network picks the maximum value across each embedding dimension. The deep averaging and max pooling architectures project the resulting patient representation onto a dense layer to capture the interrelations between semantic dimensions. The dense layer is followed by a sigmoid output. All neural network models included a dropout layer for regularization and are trained using the Adam optimizer with binary cross-entropy loss. Random search was used for tuning the neural network hyperparameters in the validation cohort [20].

\section{Model evaluation and comparisons}

The models were tuned to the highest area under the receiver operating characteristic curve (AUROC). Both neural networks and logistic regression models were examined across multiple hyperparameters (Table 1). Once the hyperparameters for each model were finalized, we combined the validation and training sets and evaluated the model in the independent test set.

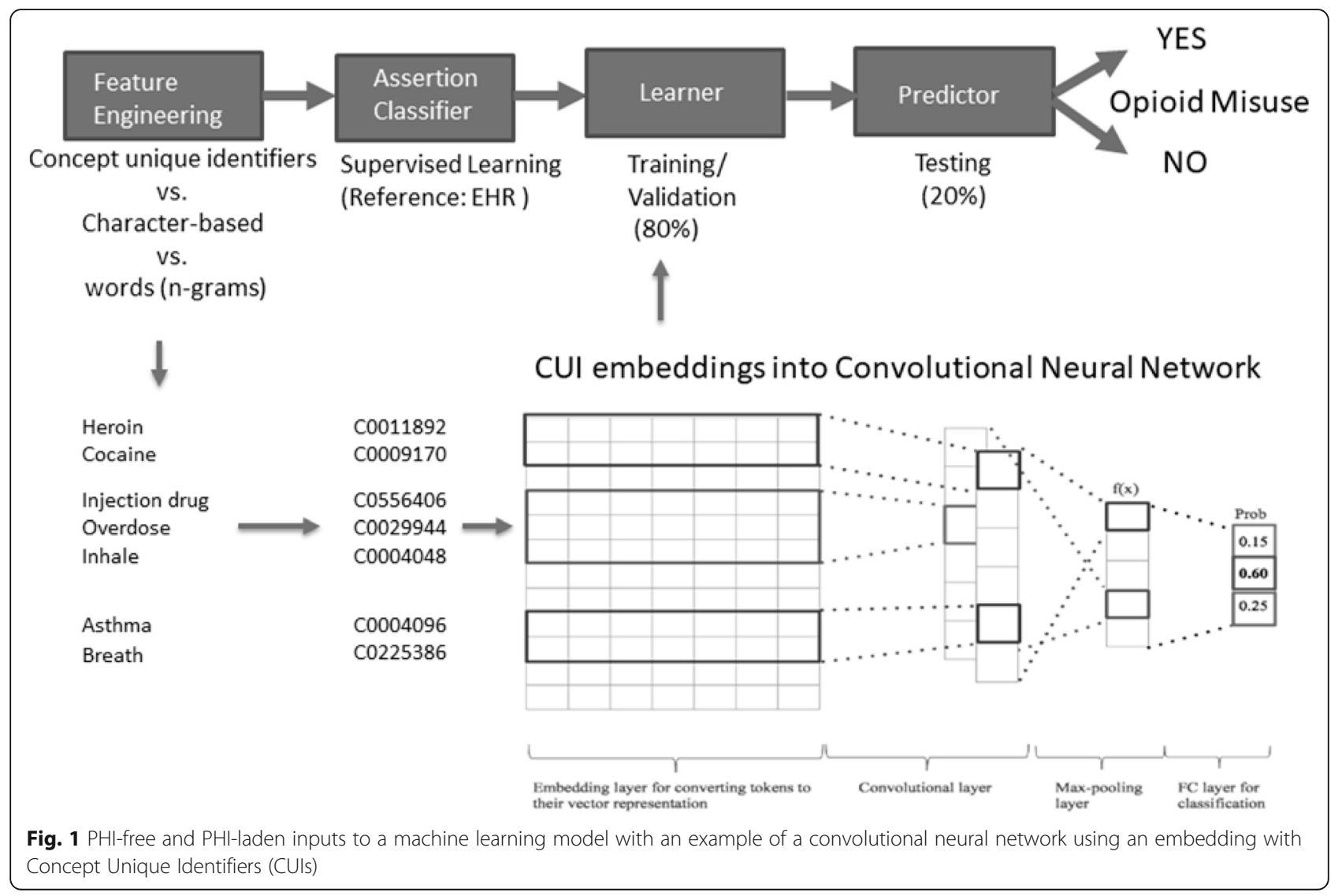


Table 1 Machine learning models with hyperparameters

\begin{tabular}{ll}
\hline Model & Hyper-parameters \\
\hline Logistic Regression-CUls & $C=1$, penalty $=$ L1, class_weight $=$ balanced \\
Logistic Regression-Words & $C=1$, penalty $=$ L1, class_weight $=$ balanced \\
Convolutional Neural Network-CUls & Filters $=1024$, Filter Size $=1$, Dropout $=0.5$, Units $=1024$, Learning Rate $=0.0001$ \\
Convolutional Neural Network-Words & Filters $=1024$, Filter Size $=3$, Dropout $=0.25$, Units $=128$, Learning Rate $=0.0001$ \\
Convolutional Neural Network-Character & Filters $=1024$, Filter Size $=11$, Dropout $=0.25$, Units $=1024$, Learning Rate $=0.0001$ \\
Deep Averaging Network-CUls & Dropout $=0.25$, Units in layer $1=2048$, Units in layer $2=512$, Learning Rate $=0.001$ \\
Deep Averaging Network-Words & Dropout $=0.75$, Units $=128$, Learning Rate $=0.001$ \\
Max Pooling Network-CUls & Dropout $=0.5$, Units $=128$, Learning Rate $=0.001$ \\
Max Pooling Network-Words & Dropout $=0.5$, Units $=128$, Learning Rate $=0.001$ \\
Deep Averaging + Max Pooling Network-CUls & Dropout $=0.5$, Units $=1024$, Learning Rate $=0.001$ \\
Deep Averaging + Max Pooling Network-Words & Dropout $=0.25$, Units $=512$, Learning Rate $=0.001$
\end{tabular}

Logistic regression's $C$ value is inverse of regularization strength, and penalty term that penalizes the loss function using different regularization techniques. Optimizer Adam is selected for all the neural networks. Units are the number of neurons in the dense layer of the neural network

Discrimination of the prediction models was evaluated using the AUROC. Goodness-of-fit was formally assessed by the Hosmer-Lemeshow test and verified visually with calibration plots. Test characteristics (sensitivity/recall, specificity, negative predictive value (NPV), precision/ positive predictive value (PPV) and macro F1 score) were provided to compare between classifiers. The DeLong et al. method was used to compare the AUROC between models [21]. For the logistic regression model, the betacoefficients of the selected features were listed to examine face validity. Analysis was performed using Python Version 3.6.5 (Python Software Foundation) and RStudio Version 1.1.463 (RStudio Team, Boston, MA). The study was approved by the Loyola University Chicago Institutional Review Board (LU \#209950). Need for consent was waived by the IRB and deemed unnecessary according to national regulations. The PHI-free models and the relevant code are publicly available in our GitHub repository (https:// github.com/AfsharJoyceInfoLab/OpioidNLP_Classifier).

\section{Results}

The data corpus of 1000 patients was comprised of 63, 301 notes, 15,651 CUI codes, and 71,987 unigrams. Classifier performance across the top neural network models for opioid misuse as well as a rule-based model and logistic regression model using CUI codes, words, and characters are displayed in Table 2.

The primary outcome was to optimize to the highest AUROC and the following were the top performing models: (1) CNN CUI; (2) CNN n-gram; (3) CNN character; (4) max pooling network CUI; (5) combined max pooling and deep averaging network CUI; (6) max pooling network n-gram; (7) combined max pooling and deep averaging network n-gram; (8) combined maxed pooling and deep averaging network CUI; (9) logistic regression n-gram; and (10) logistic regression CUI. In these top performing models, comparisons between the AUROC curves did not demonstrate any statistical differences ( $p>0.05$ for all comparisons). The AUROC curve for the CNN CUI model is displayed in Fig. 2.

Of these top performing models, only the CNN CUIs and n-grams, max pooling network CUIs and n-grams, and logistic regression CUIs fit the data well by HosmerLemeshow test $(p>0.05)$. The rule-based model did not fit the data well $(p<0.01)$. The CUI codes approach for $\mathrm{CNN}$, max pooling network and logistic regression visually fit the data best when plotted across deciles of predicted probabilities (Fig. 3).

In comparing n-gram to character-level and CUIbased features, similar test characteristics are noted for precision/PPV, recall/sensitivity, specificity, and NPV within the $\mathrm{CNN}$ and max pooling network architecture. The logistic regression CUIs had better recall/sensitivity than the logistic regression with n-grams. The CNN approach outperformed the rule-based classifier in most metrics except for recall/sensitivity and NPV. The CNN CUIs had the greatest recall/sensitivity whereas the logistic regression CUIs had the greatest precision/PPV (Table 2).

In terms of complexity, the CNN CUI model had a total number of 5,721,449 trainable parameters and the max pooling network CUI model had 4,401,257 trainable parameters. The logistic regression model selected 992 CUI code trainable parameters. L1 regularization removed zero weighted features and the final model had 21 features (Table 3). Among the selected CUI features from logistic regression, 'heroin' followed by 'victim of abuse (finding)' carried the highest beta coefficients.

\section{Discussion}

CUI code inputs to a CNN, max pooling network, and logistic regression model had the optimal performance 


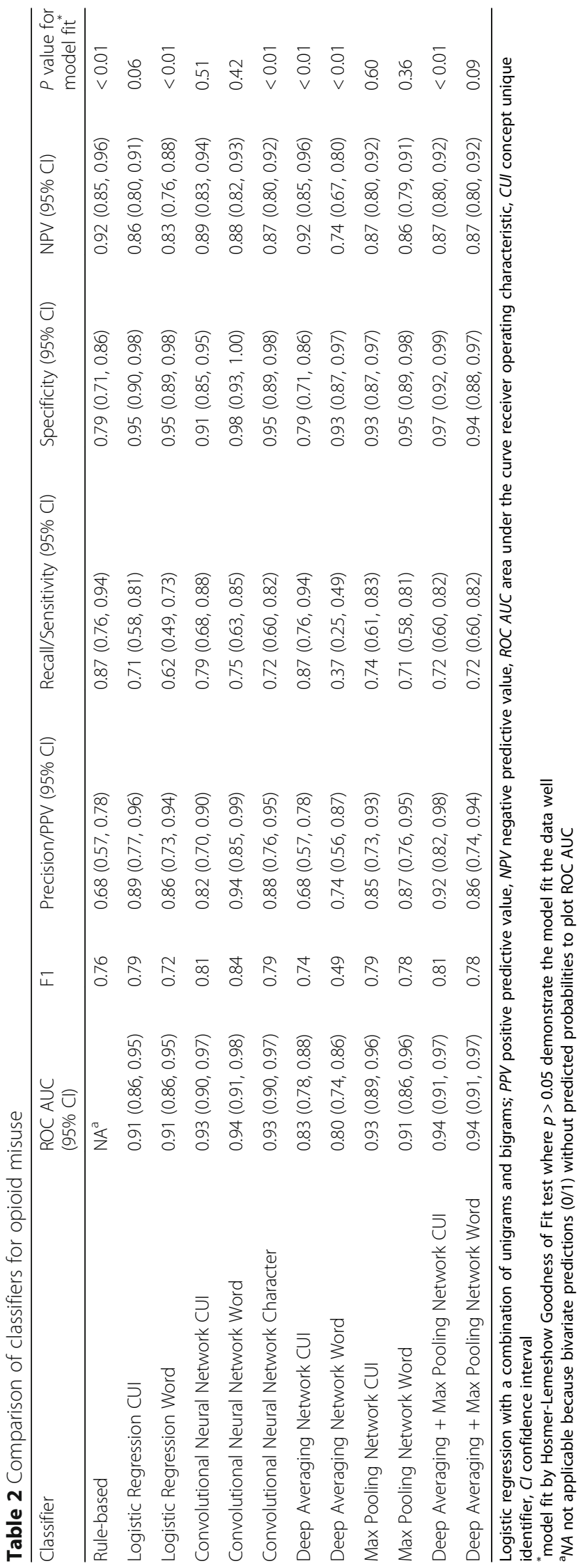




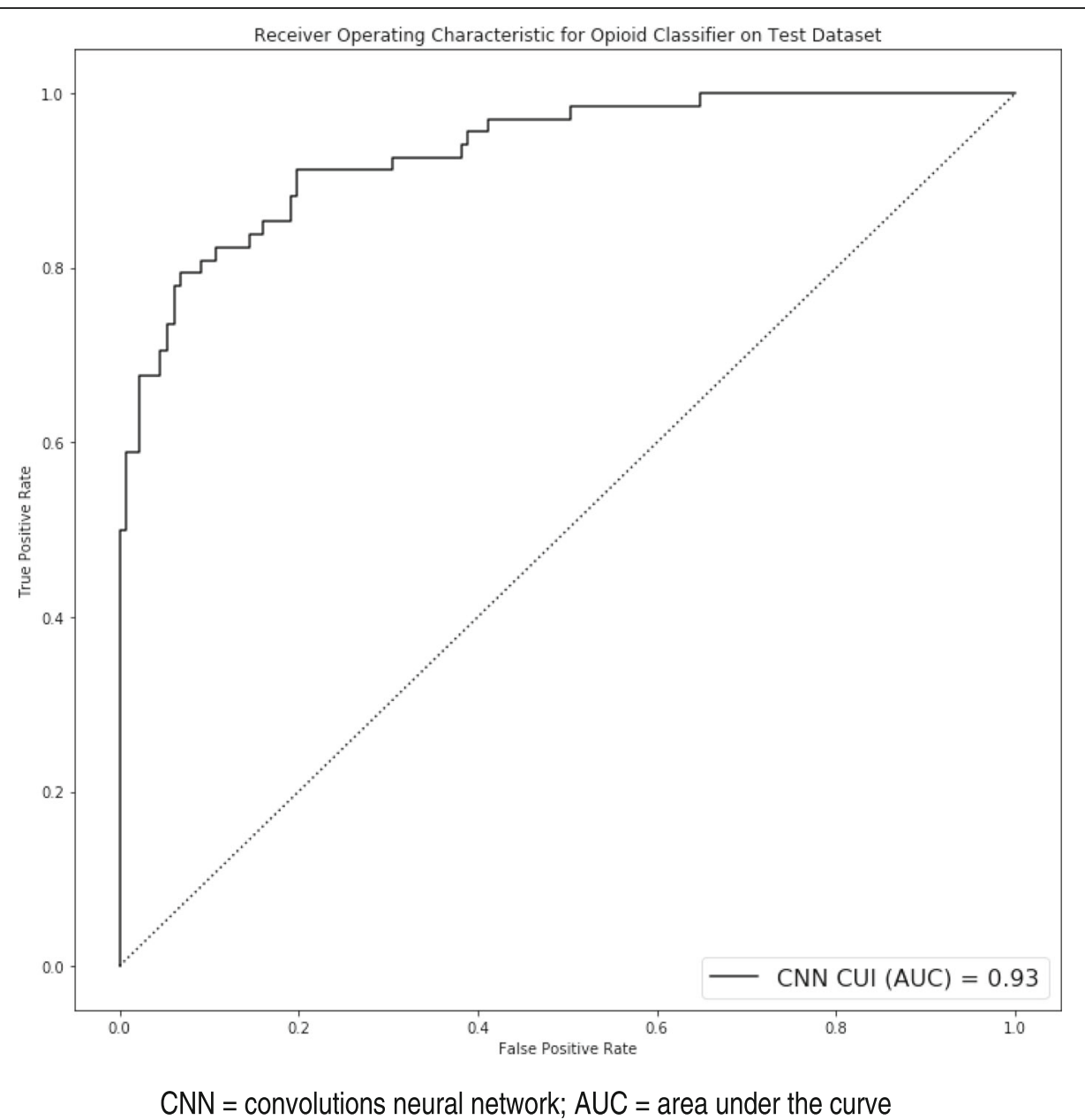

Fig. 2 Receiver operating characteristics area under the curve for convolutional neural network model using concept unique identifiers (CUI) for classification of opioid misuse. CNN = convolutions neural network; AUC = area under the curve

for an opioid misuse classifier with AUROCs greater than 0.90. CUI codes are a standardized and automated feature representation that require no domain expertise and can be extracted using off-the-shelf software. Our main finding was that PHI-free models were similar to or outperformed models containing PHI. The PHI-free neural network models ( $\mathrm{CNN}$ and max pooling network with CUI codes) were the top calibrated models with the best discrimination and are available to health systems interested in deploying these black box models with the assurance that they are compliant with the Health Insurance Portability and Accountability Act (HIPAA). The PHI-free, trained opioid misuse computable phenotypes are available on GitHub for other researchers and health systems to apply (https://github.com/AfsharJoyceInfo Lab/OpioidNLP_Classifier).

An estimated $80 \%$ of all data in EHRs reside in clinical notes $[22,23]$ and are a rich source of data, but their unstructured format makes them complex and difficult to de-identify. Recent methods for identification of the clinical notes have achieved above $90 \%$ in accuracy and F1 scores [24-26]. However, this does not constitute as fully PHI-free data and poses a barrier for health systems to share data legally. The legal requirements from HIPAA were recently highlighted in a federal class-action lawsuit making a claim that notes in the EHR of a major health system did not meet the requirements for a fully deidentified dataset [27]. Clinical notes lack common structural frameworks, contain many grammatical and spelling errors, lexical variation, and are often semantically ambiguous making de-identification difficult [28]. Methods in NLP, including concept mapping to CUI codes to produce a standard medical vocabulary, are a more effective and efficient approach for automatic semantic analyses of clinical notes [29]. We have previously shown success in using a CUI code-based approach for a NLP classifier to identify alcohol misuse and respiratory failure [30, 31].

Given the complexities of behavioral conditions like opioid misuse, very little data are available demonstrating useful computable phenotypes from CUI codes or 


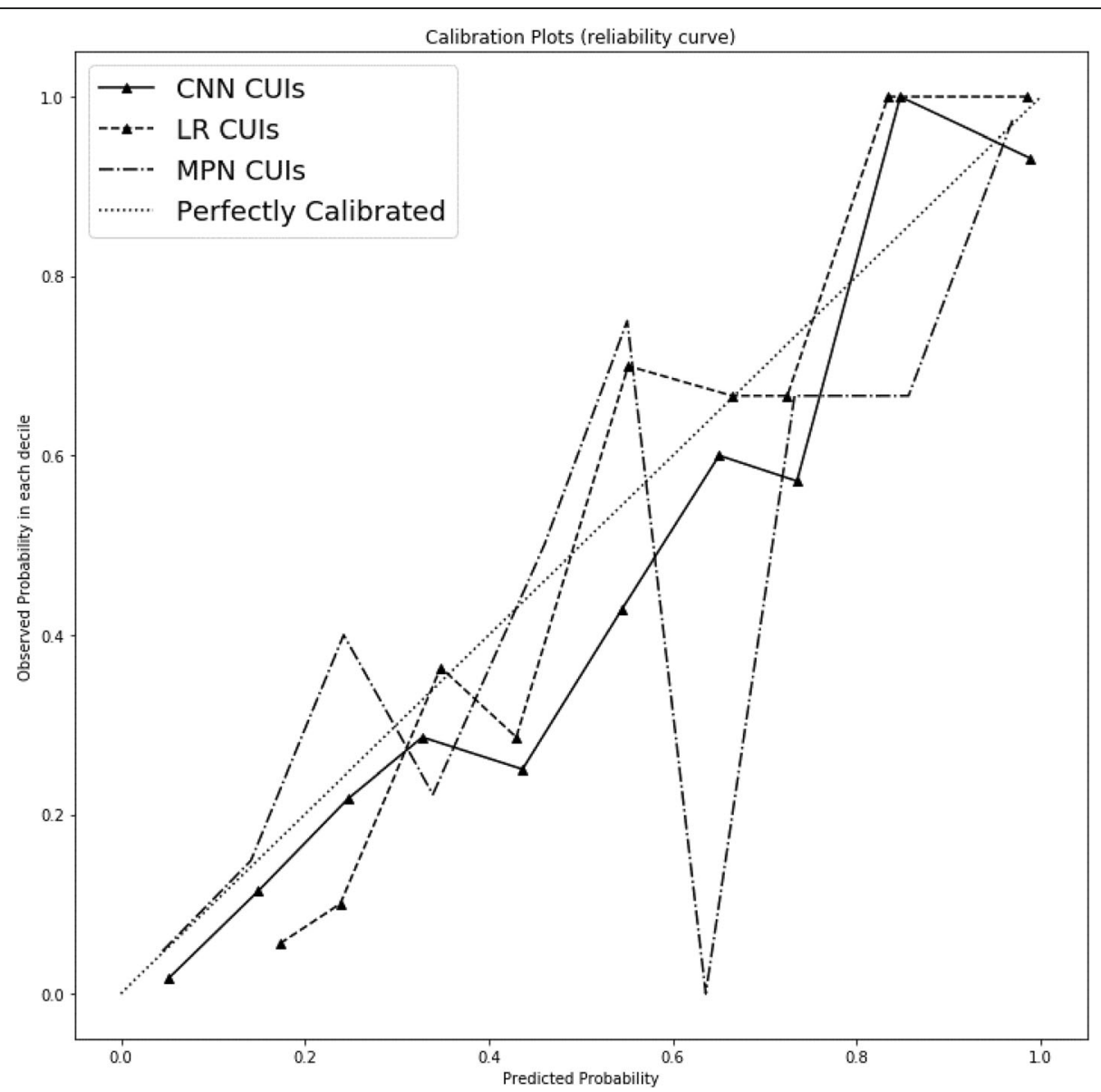

Fig. 3 Calibration plot for top performing machine learning classifiers for opioid misuse. The diagonal line represents perfect calibration between predicted probabilities that are observed ( $y$-axis) and predicted ( $x$-axis). $C N N=$ convolutions neural network; $C U l s=$ concept unique identifiers; $L R=$ logistic regression; MPN = max pooling network

character-based approaches. A systematic review of computable phenotypes for opioid misuse revealed the data used in many published algorithms are not routinely available in the EHR, use PHI-laden machine learning models, or rely solely on diagnostic billing codes [11]. To date, the best performing algorithms depend on pharmacy claims data which are not available in EHRs; therefore, are impractical to implement for providers and hospitals [32-34]. Text classification from clinical notes has demonstrated good test characteristics but have been focused in certain subtypes or specific cohorts of patients with opioid misuse and contain PHI [4, 35]. Our study using a CUI-based machine learning approach for predicting all types of opioid misuse provides a PHIfree solution and also accounts for lexical variations and semantic ambiguities. In this approach, discovery is not limited by domain knowledge or expertise, and other entity mentions outside the opioid domain may prove predictive.

Identification of opioid misuse incorporates a continuum of individuals who may occasionally use opioids for non-medical purposes to opioid use disorders, and these individuals commonly have co-occurring mental health conditions and polysubstance use [36-38]. In examining the CUI codes selected from logistic regression, this becomes apparent as clinically relevant concepts that are not explicit mentions of opioid drugs such as pain conditions, victims of abuse, and adverse events of opioid misuse are identified [39]. Also noteworthy is the logistic regression classifier identified negative features such as malignant neoplasms and acute pain conditions that are not relevant to opioid misuse. The character-based approaches for opioid misuse also proved to be useful and have similar discrimination to the logistic regression ngram model but face validity is not apparent in the more complex, neural network architecture.

Our group has previously shown advantages to opioid misuse phenotyping using transfer learning, but this paradigm is more beneficial for tasks with small sample sizes of training data [40]. In addition, transfer learning further obscures the feature extraction from more conventional neural architectures, which may be less 
Table 3 Concept Unique Identifiers (CUIs) for opioid misuse from logistic regression classifier and their $\beta$ coefficients

\begin{tabular}{|c|c|c|}
\hline $\mathrm{CUI}$ & Related text & $\beta$ coefficients \\
\hline \multicolumn{3}{|c|}{ POSITIVE FEATURES } \\
\hline C0011892 & Heroin & 16.57 \\
\hline C0344198 & Victim of abuse (finding) & 12.70 \\
\hline C0562381 & Cocaine & 4.39 \\
\hline C0025605 & Methadone & 4.19 \\
\hline C0376196 & Opiates & 4.09 \\
\hline C0001927 & Albuterol & 2.40 \\
\hline C0728755 & Dilaudid & 1.73 \\
\hline C0029944 & Drug Overdose & 1.34 \\
\hline C0030049 & Oxycodone & 1.12 \\
\hline C0150055 & Chronic pain & 0.47 \\
\hline C0040861 & Triage & 0.47 \\
\hline C1299583 & Independently able & 0.19 \\
\hline C0022742 & Knee & 0.02 \\
\hline \multicolumn{3}{|c|}{ NEGATIVE FEATURES } \\
\hline C0002903 & Anesthesia procedures & -2.08 \\
\hline C0003483 & Aorta & -1.51 \\
\hline C0006826 & Malignant Neoplasms & -1.50 \\
\hline C1272883 & Injection & -1.36 \\
\hline C0006434 & Burn injury & -0.71 \\
\hline C0020538 & Hypertensive disease & -0.42 \\
\hline C0021641 & Insulin & -0.09 \\
\hline C0004604 & Back Pain & -0.01 \\
\hline
\end{tabular}

appealing to healthcare providers. Our study utilized a training set with adequate size to achieve AUROCs above 0.90 and obviated any need for transfer learning. A future direction in this field of research involves extending pre-trained models like the Bidirectional Encoder Representations from Transformers (BERT) to very long sequences of text (e.g., thousands of tokens).

Potential limitations of our approach include a data corpus from a single hospital that may not represent the practice variations across hospitals. Further, social and behavioral determinants of health are typically limited in the EHR or not available at all [41]; however, substance use is a routinely captured data metric during intake in notes by providers, which is why we focused only on notes. The CUI approach did not prove to have appreciable differences in performance from an n-gram approach; however, a lot of the medical vocabulary may be lost during concept mapping to CUIs. This may prove to be a limitation at other health systems and potentially lead to high variance in extracted medical terms and, ultimately, performance of the classifier. For example, while some non-standard terms such as "dope" and "speedball" have CUIs, these terms may be unique to the catchment of the health system with other slang used in other regions of the US. Further misspellings of heroin and opiate/opioid, undocumented sentence structure, and other lexical variations may further contribute to the variability in mapped CUI features. Lastly, the computing resources needed to produce CUI codes and process large amounts of notes may not be readily available at other hospitals, but we have previously published a pipeline architecture for clinical data warehouses to help overcome this barrier [42].

\section{Conclusion}

PHI-free approaches for building computable phenotypes from clinical notes are needed for better surveillance and case-identification. Opioid misuse is a complex behavioral condition that requires information contained in the clinical notes, and machine learning approaches for text classification are a viable solution for case-identification. Our computable phenotypes for opioid misuse may prove useful to health systems for more accurate identification and surveillance of hospitalized patients without the risk of leaking any PHI.

\section{Abbreviations \\ PHI: Protected health information; CUI: Concept unique identifier; NLP: Natural language processing; HER: Electronic health record; ICD: International classification of disease; CTAKES: Clinical text and knowledge extraction system; UMLS: Unified medical language system; ROC AUC: Receiver operating characteristic area under the curve; PPV: Positive predictive value; NPV: Negative predictive value; CNN: Convolutional neural network; CDW: Clinical data warehouse; LUMC: Loyola University Medical Center}

\section{Acknowledgements}

We would like to thank Susan Zelisko, and Jason Boyda at Loyola's Systems Development and IT Department for providing the data from the clinical data warehouse.

\section{Authors' contributions}

All authors have read and approved the final version of this manuscript; have made a substantial contribution to the conception, design, gathering, analysis and/or interpretation of data and a contribution to the writing and intellectual content of the article; and acknowledge that they have exercised due care in ensuring the integrity of the work. Formal analysis: MA CJ, DD, $B S, K S$. Funding acquisition: MA, NSK. Investigation: BS, MA, CJ, DD. Methodology: DD, MA, BS, CJ, DD. Project administration: MA. Resources: MA, CJ, NSK. Software: MA, CJ, DD, BS. Supervision: MA, CJ, DD, ESA, NSK. Validation: MA, BS, CJ, DD, KS. Visualization: MA, BS, CJ, DD, BS. Writing original draft: $M A, B S, D D, E S A$. Writing - review \& editing: MA, CJ, DD, ESA, NSK.

\section{Funding}

This research was supported in part by the following:

(1) National Institute of Alcoholism and Alcohol Abuse grant number K23AA024503 (MA)

This fund supported the Dr. Majid Afshar's effort in performing the research and writing the manuscript.

(2) National Library of Medicine grant number R01LM012973 (DD)

This fund supported the effort in experiments by Dr. Dmitriy Dligach in neural network architectures.

(3) National Institute on Drug Abuse grant numbers R01DA04171 (NSK)

This fund provided the effort for Dr. Niranjan Karnik to share content expertise in opioid misuse.

(4) Loyola's Center for Health Outcomes and Informatics Research 
This fund paid for the server and computing resources to store the data and perform the analytics.

\section{Availability of data and materials}

The trained models generated from this study are available on the corresponding author's GitHub repository (https://github.com/ AfsharJoycelnfoLab/OpioidNLP_Classifier). The original patient data used for model training are only available from Loyola University Medical Center and Loyola University, where the data were extracted from the hospital's electronic health record (EHR) and contains patients' protected health information (PHI). These data can only be accessed for researchers who meet the legal and regulatory criteria from the institution for access to confidential data, including a data usage agreement and IRB approval with both Loyola University Chicago and Loyola University Medical Center. Requests for data may be initiated with the corresponding author (majid.afshar@lumc.edu).

\section{Ethics approval and consent to participate}

The study was approved by the Loyola University Chicago Institutional Review Board (LU \#211621). Need for patient consent was waived by the Loyola University Chicago Institutional Review Board. However, the minimal data underlying the results of this study are only available upon request due to ethical and legal restrictions imposed by the Loyola University Chicago Institutional Review Board (please see section on Data Availability).

\section{Consent for publication}

Not Applicable.

\section{Competing interests}

The authors declare they have no competing interests.

\section{Author details}

'Department of Computer Science, Loyola University Chicago, Chicago, IL, USA. ${ }^{2}$ Center for Health Outcomes and Informatics Research, Loyola University Chicago, 2160 S. First Avenue, Maywood, IL 60156, USA. ${ }^{3}$ Stritch School of Medicine, Loyola University Chicago, Maywood, IL, USA. ${ }^{4}$ Center for Multi-System Solutions to the Opioid Epidemic, American Institute for Research, Chicago, IL, USA. ${ }^{5}$ Department of Psychiatry, Rush University Medical Center, Chicago, IL, USA. 'Department of Health Informatics and Data Science, Loyola University Chicago, Maywood, IL, USA. ${ }^{7}$ Department of Medicine, Loyola University Medical Center, Maywood, IL, USA.

Received: 19 December 2019 Accepted: 22 April 2020

Published online: 29 April 2020

\section{References}

1. Demner-Fushman D, Chapman WW, McDonald CJ. What can natura language processing do for clinical decision support? J Biomed Inform. 2009:42:760-72.

2. Jones $B E$, South BR, Shao Y, et al. Development and validation of a natural language processing tool to identify patients treated for pneumonia across VA emergency departments. Appl Clin Inform. 2018;9:122-8.

3. Castro VM, Dligach D, Finan S, et al. Large-scale identification of patients with cerebral aneurysms using natural language processing. Neurology. 2017;88:164-8

4. Carrell DS, Cronkite D, Palmer RE, Saunders K, Gross DE, Masters ET, Hylan $T R$, Von Korff M. Using natural language processing to identify problem usage of prescription opioids. Int J Med Inform. 2015;84:1057-64.

5. Friedlin FJ, McDonald CJ. A software tool for removing patient identifying information from clinical documents. J Am Med Inform Assoc. 2008;15:601-10.

6. Meystre SM, Friedlin FJ, South BR, Shen S, Samore MH. Automatic deidentification of textual documents in the electronic health record: a review of recent research. BMC Med Res Methodol. 2010;10:70.

7. Meystre SM, Ferrandez O, Friedlin FJ, South BR, Shen S, Samore MH. Text de-identification for privacy protection: a study of its impact on clinical text information content. J Biomed Inform. 2014;50:142-50.

8. Ferrandez O, South BR, Shen, Friedlin FJ, Samore MH, Meystre SM. Generalizability and comparison of automatic clinical text de-identification methods and resources. AMIA Annu Symp Proc. 2012;2012:199-208.
9. National Institute of Drug Abuse. Misuse of prescription drugs. https://www. drugabuse.gov/publications/misuse-prescription-drugs/overview. Accessed 10 Oct 2019.

10. Center for Behavioral Health Statistics and Quality. 2015 National Survey on drug use and health: methodological summary and definitions. Rockville: MSAaMHS; 2016.

11. Canan C, Polinski JM, Alexander GC, et al. Automatable algorithms to identify nonmedical opioid use using electronic data: a systematic review. J Am Med Inform Assoc. 2017;24:1204-10.

12. Boscarino JA, Moorman AC, Rupp LB, et al. Comparison of ICD-9 codes for depression and alcohol misuse to survey instruments suggests these codes should be used with caution. Dig Dis Sci. 2017;62:2704-12.

13. Birman-Deych EWA, Yan Y, Nilasena DS, et al. Accuracy of ICD-9-CM codes for identifying cardiovascular and stroke risk factors. Med Care. 2005:43:480-5.

14. Weiss AJ, Bailey MK, O'Malley L, et al. Patient characteristics of opioid-related inpatient stays and emergency department visits nationally and by state, 2014: statistical brief \#224. Rockville: Healthcare Cost and Utilization Project (HCUP) Statistical Briefs; 2006.

15. Savova GK, Masanz JJ, Ogren PV, et al. Mayo clinical text analysis and knowledge extraction system (CTAKES): architecture, component evaluation and applications. J Am Med Inform Assoc. 2010;17:507-13.

16. Hughes $\mathrm{M}, \mathrm{Li}$ I, Kotoulas $\mathrm{S}$, et al. Medical text classification using convolutional neural networks. Stud Health Technol Inform. 2017;235: 246-50.

17. Iyyer M MV, Boyd-Graber J, Daume H. Deep unordered composition rivals syntactic methods for text classification. Paper presented at: Proceedings of the 53rd Annual Meeting of the Association of Computational Linguistics: volume 1, 2015; Beijing, China. 2015. https://www.aclweb.org/anthology/ P15-1162/.

18. Joulin A, Grave E, Bojanowski P, Mikolov T. Bag of tricks for efficient text classification. Paper presented at: Proceedings of the 53rd Annual Meeting of the Association of Computational Linguistics: volume 2, 2017; Valencia, Spain. 2017. https://www.aclweb.org/anthology/E17-2068/.

19. Shen D, Wang G, Wang W, et al. Baseline needs more love: on simple wordembedding-based models and associated pooling mechanisms. Paper presented at: Proceedings of the 56th Annual Meeting of the Association of Computational Linguistics: volume 1, 2018; Melbourne, Australia. 2018. https://www.aclweb.org/anthology/P18-1041.

20. Y BJaB. Random search for hyper-paramater optimization. J Mach Learn Res. 2012;13:281-305.

21. DeLong ER, DeLong DM, Clarke-Pearson DL. Comparing the areas under two or more correlated receiver operating characteristic curves: a nonparametric approach. Biometrics. 1988;44:837-45.

22. Ford $\mathrm{E}$, Carroll JA, Smith $\mathrm{HE}$, et al. Extracting information from the text of electronic medical records to improve case detection: a systematic review. J Am Med Inform Assoc. 2016;23:1007-15.

23. Meystre SM, Savova GK, Kipper-Schuler KC, et al. Extracting information from textual documents in the electronic health record: a review of recent research. Yearb Med Inform. 2008;17:128-44.

24. Jiang Z, Zhao C, He B, et al. De-identification of medical records using conditional random fields and long short-term memory networks. J Biomed Inform. 2017;75S:S43-53.

25. Stubbs A, Kotfila C, Uzuner O. Autmoated systems for the de-identification of longitudinal clinical narratives: overview of 2014 i2b2/UTHealth shared task track 1. J Biomed Inform. 2015;58:S11-9.

26. Liu Z, Tang B, Wang $X$, et al. De-identification of clinical notes via recurrent neural network and condition random field. J Biomed Inform. 2017;75S:S34-42.

27. Matt Dinerstein versus Google LLC and The University of Chicago. Class actiona complaint and demand for jury trial. United States Distrcit Court. Document accesed on 9 Sept 2019 at https://edelson.com/wp-content/ uploads/2016/05/Dinerstein-Google-DKT-001-Complaint.pdf.

28. Gonzalez-Hernandez G, Sarker A, O'Connor, et al. Capturing the patient's perspective: a review of advances in natural language processing of healthrelated text. Yearb Med Inform. 2017;26:214-27.

29. Shivade C, Raghavan P, Fosler-Lussier E, et al. A review of approaches to identifying patient phenotype cohorts using electronic health records. J Am Med Inform Assoc. 2014;21:221.

30. Afshar M, Phillips A, Karnik N, et al. Natural language processing and machine learning to identify alcohol misuse from the electronic health 
record in trauma patients: development and internal validation. J Am Med Inform Assoc. 2019;26:254-61.

31. Afshar M, Joyce C, Oakey A, et al. A computable phenotype for acute respiratory distress syndrome using natural language processing and machine learning. AMIA Annu Symp Proc. 2018;2018:157-65.

32. Hylan TR, Von Korff M, Saunders K, et al. Automated prediction of risk for problem opioid use in a primary care setting. J Pain. 2015;16:380-7.

33. Smith RC, Frank C, Gardiner JC, et al. Pilot study of a preliminary criterion standard for prescription opioid misuse. Am J Drug Alcohol Abuse. 2010;19: 523-8.

34. Wang Y, Chen ES, Pakhomov S, et al. Automated extraction of substance use information from clinical texts. AMIA Annu Symp Proc. 2015;2015:2121-30.

35. Lingeman JM, Wang $P$, Becker, et al. Detecting opioid-related aberrant behavior using natural language processing. AMIA Annu Symp Proc. 2017; 2018:1179-85.

36. Edlund MJ, Steffick D, Hudson T, et al. Risk factors for clinically recognized opioid abuse and dependence among veterans using opioids for chronic non-cancer pain. Pain. 2007;129:355-62.

37. Doran KM, Rahai N, McCormack RP, et al. Substance use and homelessness among emergency department patients. Drug Alcohol Depend. 2018;188: 328-33.

38. Lee C, Sharma M, Kantorovich S, Brenton A. A predictive algorithm to detect opioid use disorder: what is the utility in a primary care setting? Health Serv Res Manag Epidemiol. 2018:5:2333392817747467.

39. Grigsby TJ, Howard JT. Prescription opioid misuse and comorbid substance use: past 30-day prevalence, correlates and co-occurring behavioral indicators in the 2016 national survey on drug use and health. Am J Addict. 2019;28:111-8

40. Dligach D, Afshar M, Miller T. Toward a clinical text encoder: pretraining for clinical natural language processing with applications to substance misuse. J Am Med Inform Assoc. 2019;26:1272.

41. Arons A, DeSilvey S, Fichtenberg C, et al. Documenting social determinants of health-related clinical activities using standardized medical vocabularies. JAMIA Open. 2019;2:81-8.

42. Afshar M, Dligach D, Sharma B, et al. Development and application of a high throughput natural language processing architecture to convert all clinical documents in a clinical data warehouse into standardized medical vocabularies. J Am Med Inform Assoc. 2019;26:1364-9.

\section{Publisher's Note}

Springer Nature remains neutral with regard to jurisdictional claims in published maps and institutional affiliations.

Ready to submit your research? Choose BMC and benefit from:

- fast, convenient online submission

- thorough peer review by experienced researchers in your field

- rapid publication on acceptance

- support for research data, including large and complex data types

- gold Open Access which fosters wider collaboration and increased citations

- maximum visibility for your research: over $100 \mathrm{M}$ website views per year

At $\mathrm{BMC}$, research is always in progress.

Learn more biomedcentral.com/submissions 